\title{
Clinical Study on Treatment of Vascular Aging in Patients with Type 2 Diabetes Complicated by Hypertension with Combination of Traditional Chinese Medicine and Western Medicine
}

$\mathrm{Bin} \mathrm{Ju}$

The Third People's Hospital of Taizhou, Taizhou 225321, Jiangsu Provinces, China

\begin{abstract}
Objective: To explore the clinical effect of combination of traditional Chinese medicine and western medicine in treatment of vascular aging in patients with type 2 diabetes complicated by hypertension. Methods: Ninety patients with type 2 diabetes complicated by hypertension admitted to our hospital from May 2016 to August 2019 were selected as research objects. They were randomly divided into control group and observational group, with 45 cases each. Control group was given amlodipine besylate combined with metformin hydrochloride. On the basis of control group, observational group was given combination of TCM syndrome differentiation. Blood glucose, blood pressure and blood lipids before and after 14 days of treatment were compared between two groups. Results: Blood glucose, blood pressure and lipid indexes after treatment were lower than before treatment in both groups; observational group was lower than control group and the difference was statistically significant $(\mathrm{P}<0.05)$. Conclusion: Combination of traditional Chinese medicine and Western medicine could lower blood glucose and blood pressure indexes, control blood lipids and delay blood vessel aging in patients with type 2 diabetes complicated by hypertension, it is worthy of clinical popularization.
\end{abstract}

Keywords: Type 2 diabetes complicated by hypertension; Traditional Chinese medicine and western medicine treatment; Vascular aging

Publication date: July, 2019

Publication online: 10 July 2019
*Corresponding author: Bin Ju, jubin2010@sina.com

Diabetes is a group of endocrine and metabolic syndromes due to absolute or relative lack of insulin in patient body, which lead to metabolic disorders of sugar, fat, and protein. Clinically, it is categorized into 4 types: type 1 diabetes, type 2 diabetes, special type diabetes and gestational diabetes. Type 2 diabetes refers to patients with insulin resistance accompanied with relative insulin deficiency or apparent lack of insulin. These patients are mainly middle-aged and elderly people, with most of whom are obese and have varying degrees of hypertension. Type 2 diabetes complicated by hypertension can affect blood vessel to varying degrees and cause blood vessel aging ${ }^{[1]}$. The objective of this study was to explore the clinical effect of combination of traditional Chinese medicine and western medicine in treatment of vascular aging in patients with type 2 diabetes complicated by hypertension. Report is as follows.

\section{Materials and methods}

\subsection{General information}

Approval was obtained from Medical Ethics Committee of our hospital. Ninety patients with type 2 diabetes complicated by hypertension who were admitted to our hospital from May 2016 to August 2019 were selected as research objects. They were randomly divided into control group and observational group, with 45 cases in each group. Control group consisted of 24 males and 21 females; aged 57-82 years old, mean age $(68.29 \pm 9.53)$ years old; disease duration 2-10 years, mean disease 
duration $(5.38 \pm 2.76)$ years. Observational group consisted of 25 males and 20 females; aged 57-83 years old; mean age $(68.33 \pm 9.47)$ years old; disease duration $1-10$ years, mean disease duration $(5.29 \pm 2.82)$ years. General information of two groups of patients was compared, there was no statistically significant difference $(P>0.05)$; data comparison could be applied.

\subsection{Inclusion criteria}

(1) Inclusion criteria: patient who met the diagnostic criteria for high blood pressure and type 2 diabetes as outlined in "Chinese Basic Guideline for Management of Hypertension" ${ }^{[2]}$ and "Basic Guideline for Diagnosis and Treatment of Type 2 Diabetes" ${ }^{\text {"[3] }}$; no allergy history to drug used in this study; signed informed consent from patient and family members. (2) Exclusion criteria: patient with severe abnormalities in liver and kidney function; patient with communication disorder; patient with mental illness; patient complicated with other serious primary disease.

\subsection{Methods}

Two groups of patients were given diet and exercise intervention. Appropriate calorie and structure intake control was applied in diet course of patients, and appropriate physical exercises were developed for patients.

\subsubsection{Control group}

Western medicine treatment: oral dose of amlodipine besylate was given, $5 \mathrm{mg} /$ tablet, once/day; oral dose of metformin hydrochloride was given, $0.5 \mathrm{~g}$ / tablet, 3 times/day.

\subsubsection{Observational group}

Combination of traditional Chinese medicine and western medicine for treatment. On the basis of control group, traditional Chinese medicine syndrome differentiation and treatment was applied. (1)Lung and stomach dryness and heartiness (8 cases): evidence of frequent thirst, dry mouth, frequent urination, frequent hunger and diet, thin body, and dry stool. Red tongue, yellow tongue coat, smooth and rapid pulse. Treatment was based on moistening of lungs and stomach, hydration promotion and thirst-quenching. Prescription was thirst-quenching and hydration-promoting decoction (Huanglian, Trichosanthin, Shengdi, Xuanshen, Ophiopogon, Yuzhu, Zhimu, Lugen). (2)Qi and yin deficiency (10 cases): evidence of dry mouth, dry lips, frequent or turbid urination, fatigue, dizziness, and waist and knee faintness. Red tongue, thin tongue coat, weak or fine pulse. Treatment was based on $q i$ and yin nourishment, and spleen and kidney nourishment. Prescription was fluid-nourishing and pancreasnourishing decoction (Astragalus, Zhimu, Pueraria, Schisandra, yam, Shengshudi, dogwood, wolfberry). (3) Yin and yang deficiency (9 cases): frequent urination, frequent water consumption especially at night, dry mouth, dry face, waist and knees faintness, impotence, cold limbs, puffy feet, pale and fat tongue, thin and white tongue coat, and weak pulse. Treatment was based on kidney nourishment via yang supplementation. Prescription was Jinkui kidney-nourishing pill (Fupian, cinnamon powder, Shengshudi, yam, dogwood, Fuling, Pachyderma, and Zexie); (4)Blood stasis and impediment type (18 cases): dry tongue and reduced water consumption, limb numbness, hemiplegia, or chest pain. Tongue may have ecchymosis, and pulse may be weak and impeded. Treatment was based on nourishment of $q i$ and yin, and blood circulation promotion. Prescription was yang-supplementing and hydration-promoting decoction (Astragalus, Angelica, Chuanxiong, Chiba, Dilong, Shengdi, Maidong, Xuanshen). Prescriptions above were decocted, dregs were discarded, $200 \mathrm{~mL}$ of decoction was retained and $100 \mathrm{~mL}$ was taken in morning and evening, 1 dose/day. Patients in both groups received treatment for 14 days.

\subsection{Evaluation indexes}

(1) Before and 14 days after treatment, fingertip blood was collected from both groups of patients. Fasting blood glucose and blood glucose at 2 hours after meal of both patient groups were measured using Johnson's blood glucose meter. Blood pressure was measured using OMRON upper arm blood pressure monitor. (2) Before treatment and 14 days after treatment, $5 \mathrm{~mL}$ of fasting venous blood was collected from two groups of patients for measurement of blood lipids: triglyceride (TG), high-density lipoprotein (HDL-C), and lowdensity lipoprotein (LDL-C).

\subsection{Statistical method}

SPSS 18.0 software was used for data processing. Quantitative data was expressed in $(\bar{x} \pm s)$. Independent samples $t$ test was applied for comparison between groups, and paired sample t test was applied for comparison within group. Qualitative data was expressed in the form of percentage. $\chi^{2}$ test was applied and $P<0.05$ indicated significant statistical difference. 


\section{Results}

\subsection{Blood glucose and blood pressure}

Blood glucose and blood pressure indexes after treatment were lower than before treatment for both groups of patients. Observational group was lower than control group, and the difference was statistically significant $(P<0.05)$. (Table 1).

Table 1. Comparison of blood glucose and blood pressure between two groups $(\bar{x} \pm \mathrm{s})$

\begin{tabular}{|c|c|c|c|c|c|}
\hline Time & Group & $\begin{array}{c}\text { Fasting blood } \\
\text { glucose }(\mathrm{mmol} / \mathrm{L})\end{array}$ & $\begin{array}{c}\text { Blood glucose at } 2 \mathrm{~h} \text { after } \\
\text { meal }(\mathrm{mmol} / \mathrm{L})\end{array}$ & $\begin{array}{c}\text { Systolic pressure } \\
(\mathbf{m m H g})\end{array}$ & $\begin{array}{c}\text { Diastolic } \\
\text { pressure(mmHg) }\end{array}$ \\
\hline \multirow{4}{*}{$\begin{array}{c}\text { Before } \\
\text { treatment }\end{array}$} & Control $(n=45)$ & $15.12 \pm 1.41$ & $16.12 \pm 1.65$ & $162.08 \pm 10.27$ & $105.47 \pm 6.53$ \\
\hline & $\begin{array}{l}\text { Observational } \\
\qquad(\mathrm{n}=45)\end{array}$ & $15.07 \pm 1.53$ & $16.24 \pm 1.59$ & $162.59 \pm 10.14$ & $105.52 \pm 6.44$ \\
\hline & $t$ & 0.168 & 0.367 & 0.247 & 0.038 \\
\hline & $P$ & 0.867 & 0.715 & 0.805 & 0.970 \\
\hline \multirow{4}{*}{$\begin{array}{c}\text { After } \\
\text { treatment }\end{array}$} & Control $(n=45)$ & $12.25 \pm 1.33^{\mathrm{a}}$ & $12.31 \pm 1.69^{\mathrm{a}}$ & $144.69 \pm 9.16^{\mathrm{a}}$ & $91.17 \pm 4.95^{\mathrm{a}}$ \\
\hline & $\begin{array}{l}\text { Observational } \\
\qquad(\mathrm{n}=45)\end{array}$ & $5.27 \pm 0.51^{\mathrm{a}}$ & $6.21 \pm 0.63^{\mathrm{a}}$ & $123.27 \pm 8.58^{\mathrm{a}}$ & $82.47 \pm 4.16^{\mathrm{a}}$ \\
\hline & $t$ & 34.304 & 23.675 & 11.947 & 9.419 \\
\hline & $P$ & 0.000 & 0.000 & 0.000 & 0.000 \\
\hline
\end{tabular}

Note: Comparison with before treatment within the same group, ${ }^{\mathrm{a}} \mathrm{P}<0.05$

\subsection{Blood lipids}

Blood lipids after treatment were lower than before treatment in both groups of patients. Observational group was lower than control group, and the difference was statistically significant $(P<0.05)$. (Table 2).

Table 2. Comparison of blood lipid levels between two groups $(\bar{x} \pm \mathrm{s}, \mathrm{mmol} / \mathrm{L})$

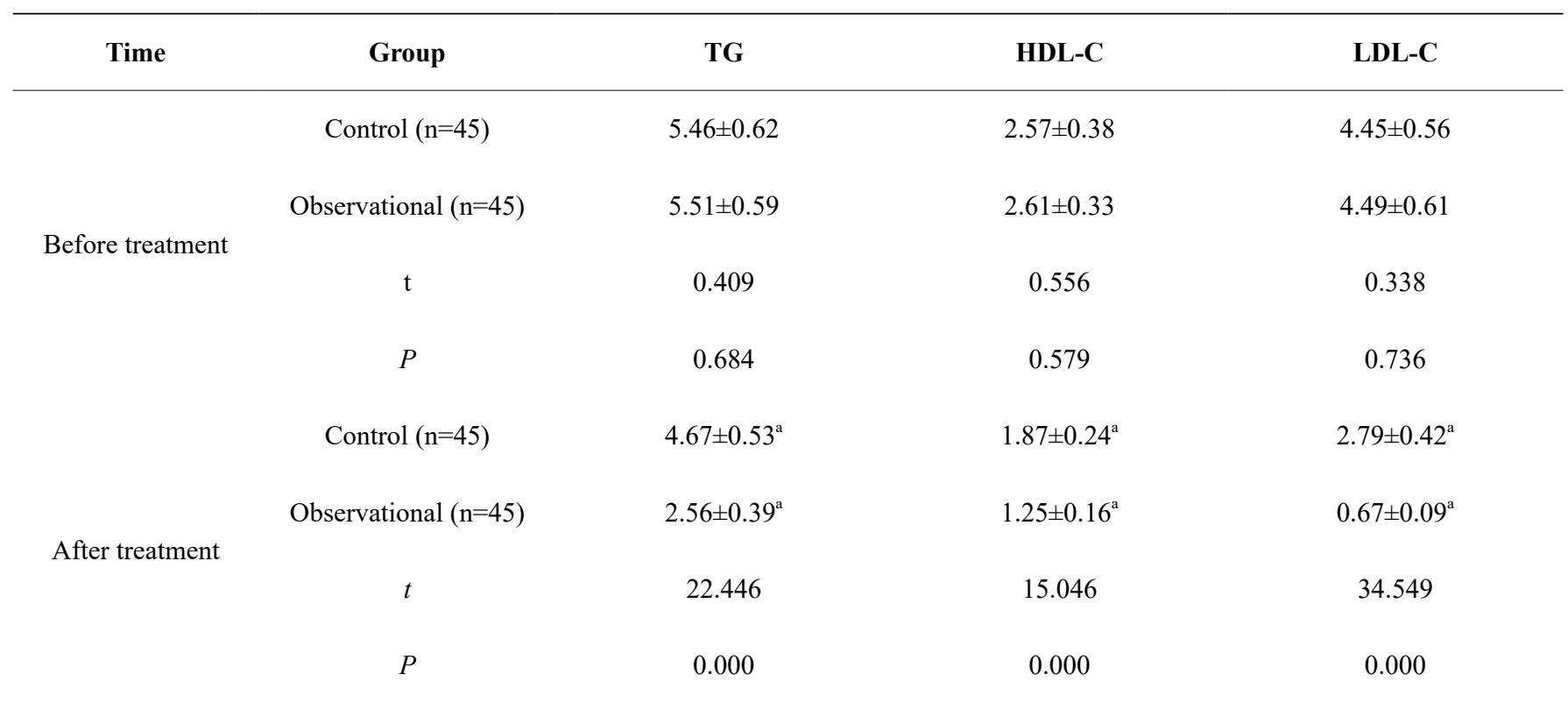

Note: Comparison with before treatment within the same group, ${ }^{a} P<0.05$ 


\section{Discussions}

Type 2 diabetes is due to ineffective function of insulin (less binding to receptor). It is a life-long disease which relies on continued treatment of blood sugar control and active treatment of diabetes complications, which include high blood pressure - to prevent vicious cycle caused by diabetes $^{[4]}$.

The results of this study showed that blood glucose, blood pressure and blood lipid indexes after treatment were lower than before treatment in two groups of patients, and observational group was lower than that of control group. This showed that combination of traditional Chinese medicine and western medicine could lower blood glucose and blood pressure indexes, and control blood lipids and delay blood vessel aging in patients with type 2 diabetes complicated by hypertension. With the advancement of medicine, physical examination of cardiovascular and cerebrovascular diseases e.g. blood lipids, blood sugar and blood pressure, can reflect aging degree of blood vessel. Vascular aging is a phenomenon in which blood vessels gradually lose their original function as the body ages, which leads to increased arterial stiffness and increased pulse wave transmission rate. Blood vessel aging will lead to smaller inner diameter of blood vessel and slower blood flow rate. Patient will experience symptoms such as dizziness, headache, decreased memory, limb numbness, and chest tightness ${ }^{[5-6]}$.

Metformin drugs have the capability to lower glucose output from liver, and help muscle cells, fat cells, and liver in capturing more glucose from blood which thereby lower blood sugar level. Amlodipine can enlarge peripheral arterioles, normal and ischemic coronary arteries and coronary arteries; which reduce peripheral resistance (post-load), reduce myocardial energy consumption and oxygen demand, and increase myocardial oxygenation in patients with coronary spasm (variant angina pectoris) ${ }^{[7-8]}$. Traditional Chinese medicine believes that diabetes is attributed to both internal and external factors. Internal factor is yin deficiency of body, while external factors include dysphagia, emotional disorder, excessive exertion or heartiness, which cause endogenous heat, loss of yin and subsequent frequent thirst, diet, urination and thin body. The main pathological changes are yin deficiency and heartiness. Main treatments are based on clearing and moistening of lungs and stomach, nourishment of $q i$ and yin, nourishment of kidney by yang supplementation and promotion of blood circulation. Combination of traditional Chinese medicine and western medicine leads to more effective treatment ${ }^{[9-10]}$.

In summary, treatment of patients with type 2 diabetes complicated by hypertension with combination of traditional Chinese medicine and western medicine could reduce blood glucose and blood pressure indicators, control blood lipids and delay blood vessel aging. It is worthy of clinical popularization.

\section{References}

[1] Cai YW, Li JH, Ye SS, et al. Relationship between intestinal microbe distribution and estimated glomerular filtration rate in elderly patients with type 2 diabetes complicated with hypertension[J]. Zhejiang Medical Journal, 2019, 41(19): 2067-2071.

[2] Revision Committee of "China Basic Guideline for Management of Hypertension". China Basic Guideline for Management of Hypertension (2014 Revision) [J]. Chinese Journal of Hypertension, 2015, 30(1): 24-30.

[3] Chinese Medical Association, Chinese Medical Association Journal, General Medical Branch of Chinese Medical Association, et al. Basic Guideline for Diagnosis and Treatment of Type 2 Diabetes (Practical Version 2019) [J]. Chinese Journal of General Physicians, 2019, 18(9): 810-818.

[4] Li BX, Li Y, Liu LS. Correlation between hypertension and hyperuricemia and type 2 diabetes [J]. Journal of Cardiopulmonary Vascular Disease, 2019, 38(8): 830-832.

[5] Jiang M, Wang PL, Yang J, et al. Analysis of vascular aging indexes in patients with type 2 diabetes and hypertension $[\mathrm{J}]$. Journal of Integrated Traditional Chinese and Western Medicine Cardiovascular and Cerebrovascular Disease, 2018, 16(16): 2289-2292.

[6] Cheng X, Qian MW, Zou XM, et al. Clinical characteristics of patients with type 2 diabetes and hypertension [J]. Chinese Journal of Clinical Health Care, 2019, 22(4): 530-533.

[7] Gao YB, Wang F. Effect of Tongxinluo Capsule on Hypertension and Type 2 Diabetes on Vascular Endothelial Function and Oxidative Stress in Patients [J]. Modern Journal of Integrated Traditional Chinese and Western Medicine, 2019, 28(5): 529-532.

[8] Diao H. Treatment of elderly essential hypertension with type 2 diabetes by integrated traditional Chinese and western medicine $[\mathrm{J}]$. Jilin Journal of Traditional Chinese Medicine, 2019, 39(3): 341-343.

[9] Huo MY, Guo YF. Effects of blood glucose-lowering and blood pressure-lowering decoctions on adipokines and cytokines in patients with type 2 diabetes complicated with hypertension $[\mathrm{J}]$. Journal of Hunan University of Traditional Chinese Medicine, 2019, 39(08): 998-1002.

[10] Su J, Xu H, Yang JQ. Blood lipid and blood glucose metabolism in patients with type 2 diabetes and hypertension and their relationship with course of hypertension [J]. Guizhou Medical Journal, 2019, 43(10): 1564-1565. 